\title{
Cavitation Mediated Rat Lung Bioeffects From Diagnostic Ultrasound
}

\author{
C. K. Holland ${ }^{\mathrm{a}}$, R. A. Roy ${ }^{\mathrm{b}}$, P. W. Biddinger ${ }^{\mathrm{c}}$, C. J. Disimile ${ }^{\mathrm{a}}$ and C. Caywood ${ }^{\mathrm{d}}$ \\ ${ }^{a}$ Department of Biomedical Engineering, University of Cincinnati, Cincinnati, Ohio 45267-0761 USA \\ ${ }^{b}$ Department of Aerospace and Mechanical Engineering, Boston University, Boston, 02215 MA USA \\ ${ }^{c}$ Department of Pathology, University of Cincinnati, Cincinnati, Ohio USA \\ ${ }^{d}$ Department of Laboratory Animal Medicine, University of Cincinnati, Cincinnati, Ohio USA
}

\begin{abstract}
Several animal models have exhibited petechial hemorrhage in lung within the current output limit of clinical diagnostic ultrasound systems. The experimental threshold in acoustic pressure for damage is approximately inversely dependent on the square root of frequency [1], which is consistent with a cavitational mechanism of damage. To elucidate the mechanism of damage, rat lungs were simultaneously exposed or sham exposed in vivo to 6-MHz pulsed Doppler ultrasound and interrogated with a confocally aligned 30-Mz active cavitation detector (ACD) [2]. At the termination of ultrasound or sham exposure, each animal was immediately euthanized with sodium pentobarbital $(200 \mathrm{mg} / \mathrm{kg} \mathrm{IP})$ and lung tissues were removed intact, inflated with buffered formalin, and examined by a pathologist who was blinded to the exposure conditions. Histologically, damage was observed in the lungs exposed to ultrasound as extravasation of erythrocytes into the alveolar spaces. The received ACD signal was analyzed for the presence of increased scattering and radiated noise from inertial cavitation. The variance of the sequentially obtained ACD received pulses correlated with the damage in the exposed lungs. We conclude that inertial cavitation mediated the damage from diagnostic ultrasound.
\end{abstract}

\section{INTRODUCTION}

Although no adverse effects on patients or clinicians due to diagnostic ultrasound exposure has been documented to date, several animal models have exhibited a threshold for petechial hemorrhage in lung that is within the acoustic output levels typical of currently available diagnostic ultrasound systems [1]. Inertial cavitation has been discussed as a possible damage mechanism, though other acousto-mechanical effects that act directly on the lung surface could work in concert with cavitation. Several investigators have provided evidence for the existence of cavitation due to exposure to diagnostic ultrasound in vivo [1]. Our specific aim was to determine the relationship between cavitation and the development of petechial hemorrhage in rat lung due to exposure to pulsed Doppler ultrasound.

\section{APPARATUS, EXPERIMENTAL PROCEDURE AND RESULTS}

A clinical ultrasound scanner, an Advanced Technical Laboratories (Seattle, WA, USA) HDI 3000 was utilized to initiate lung damage and cavitation in 200 g Sprague Dawley rats at an MI of 1.8 onscreen. An L10-5 linear array was initially driven by the ATL system in B-mode for alignment $(\mathrm{MI}<0.1)$ and then in pulsed Doppler mode $(\mathrm{PRF}=1250 \mathrm{~Hz})$ for the cavitation and damage studies. The 6-MHz Doppler pulse had a $1.5 \mathrm{~mm}$ spatial pulse. The $30-\mathrm{MHz}$ active cavitation detector (ACD) was employed to detect inertial cavitation (sometimes referred to as transient cavitation owing to its' sporadic nature) [2]. The ACD and ATL L10-5 transducers were aligned $90^{\circ}$ relative to one another and their confocal regions were placed at the pleural surface of the anesthetized rat in a $37 \mathrm{C}$ water-filled exposure tank. A LeCroy 9350CL 500 $\mathrm{MHz}$ digital oscilloscope was used in sequential mode to capture the initial 50 received ACD pulses for each exposure.

Seven rats were anesthetized with Ketamine (87 $\mathrm{mg} / \mathrm{kg})$ and Xylazine $(13 \mathrm{mg} / \mathrm{kg})$ IP according to a protocol approved by the institutional animal care and use committee. Hair was gently shaved and depilated form the thoracic and axillary regions. Three rats were sham exposed and four rats were exposed to $6.0-\mathrm{MHz}$ pulsed Doppler for $2.0 \mathrm{~min}$ and immediately euthanized with sodium pentobarbital $(200 \mathrm{mg} / \mathrm{kg})$ IP. The chest was opened and the contents removed en bloc. The lungs were inflated with $10 \%$ buffered formalin and immersed until fixation. The lungs were processed for routine histology, the resulting slides were stained with hematoxylin-eosin and the damage was assessed by a pathologist blinded to the exposure conditions.

Shown in Fig. 1 is the histological appearance of petechial hemorrhage noted in rat lung exposed to 6.0 $\mathrm{MHz}$ pulsed Doppler ultrasound at an MI of 1.8. Three of the four insonified rats exhibited distinct 
cavitation events, each having a characteristic of the digital oscilloscope trace shown in Fig. 2. The received ACD amplitude is a direct indication of the individual bubble or cavitation-zone size variation with time. The bubble complex exists for a period of approximately $1 \mu \mathrm{s}$, the same as the Doppler pulse duration. The mean received ACD amplitude and the variance was determined for each $5 \mu$ s received pulse for the entire 50-segment sequence. Because inertial cavitation tends to be sporatic in space and time, the variance of the 50 segments can be used as an indicator of cavitation dose. The mean received ACD amplitude for one rat that was exposed to pulsed Doppler ultrasound is shown in Fig. 3 and results for both received amplitude and variance for all 7 rats are displayed in Table 1.

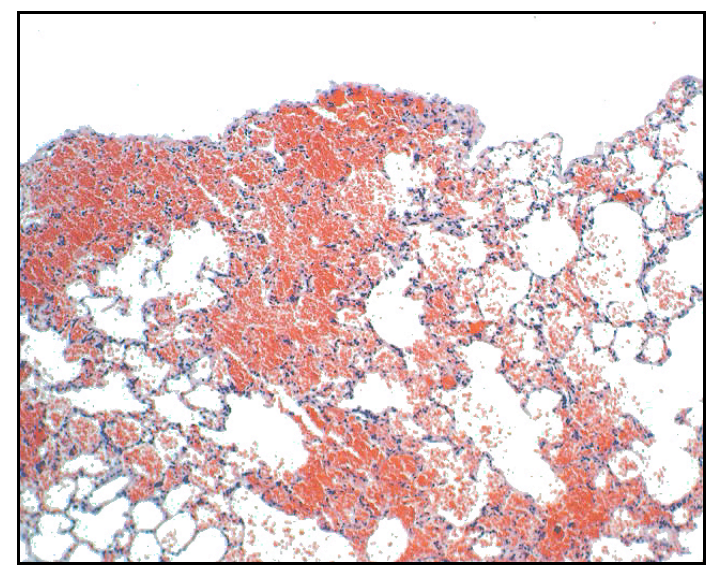

FIGURE 1. Subpleural petechial hemorrhage in rat lung parenchyma exposed to $6-\mathrm{MHz}$ pulsed Doppler ultrasound at an MI of 1.8. Note extravasation of erythrocytes into the alveolar spaces.

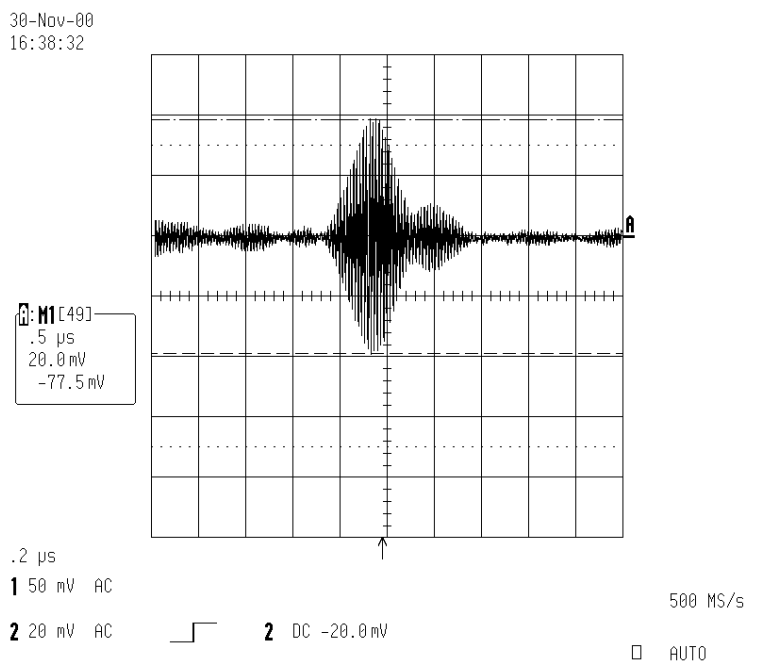

FIGURE 2. 30-MHz ACD received pulse from rat lung exposed to $6.0 \mathrm{MHz}$ pulsed Doppler ultrasound an MI of 1.8 .

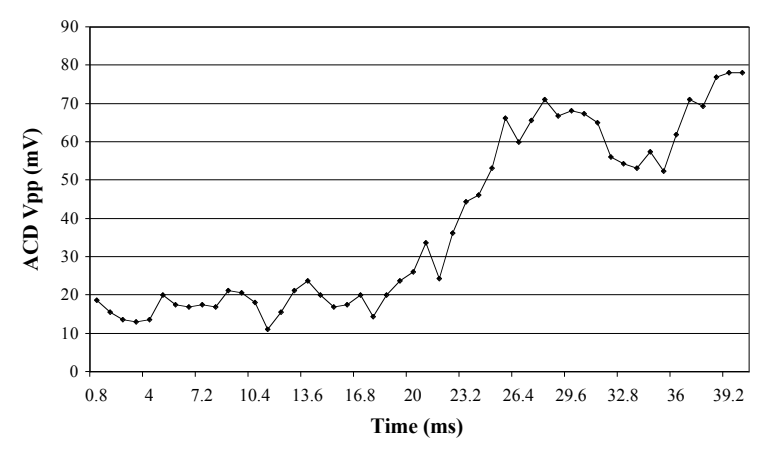

FIGURE 3. Mean $30-\mathrm{MHz}$ ACD received pulse amplitude (Vpp) in each of 50 segments from rat lung exposed to $6.0 \mathrm{MHz}$ pulsed Doppler ultrasound.

Table 1. Active Cavitation Detector mean received amplitude and variance over 50 received pulses tabulated as a function of exposure conditions.

\begin{tabular}{ccrrr}
\hline $\begin{array}{c}\text { Rat } \\
\text { No. }\end{array}$ & $\begin{array}{c}\text { Exposure } \\
\text { (M.I.) }\end{array}$ & Damage? & $\begin{array}{c}\text { ACD } \\
<\mathrm{Vpp}>\end{array}$ & $\begin{array}{c}\text { ACD } \\
\text { Variance }\end{array}$ \\
\hline 266 & Sham & $\mathrm{N}$ & 20.9 & 31.4 \\
267 & Sham & $\mathrm{N}$ & 27.1 & 48.3 \\
268 & Sham & $\mathrm{N}$ & 12.9 & 6.4 \\
269 & 1.8 & $\mathrm{Y}$ & 52.8 & 3236.2 \\
270 & 1.8 & $\mathrm{Y}$ & 38.6 & 530.0 \\
271 & 1.8 & $\mathrm{~N}$ & 20.1 & 22.7 \\
272 & $0.4-1.2$ & $\mathrm{Y}$ & 28.2 & 254.0 \\
\hline
\end{tabular}

\section{DISCUSSION AND CONCLUSIONS}

The exposed rats with lung damage exhibited much larger variance in the ACD received amplitude than the those animals with no damage (both exposed and sham). This indication of cavitational activity correlated with damage in the exposed lung. Monitoring the "running variance" (averaging over 1 to 10 segments) might be a more sensitive indicator of the onset of cavitation.

\section{ACKNOWLEDGMENTS}

This research was supported by National Institutes of Health grant R29 HL58761-02.

\section{REFERENCES}

1. AIUM, J. Ultrasound Med. 19, 97-108 (2000).

2. R. A. Roy, S. Madanshetty, and R. E. Apfel, J. Acoust. Soc. Am. 20, 2451-2455 (1990). 